\title{
Uniqueness of polynomial and differential monomial
}

\author{
Harina P. Waghamore \\ Department of Mathematics, \\ Jnanabharathi Campus, \\ Bangalore University, India \\ email: harinapw@gmail.com, \\ harina@bub.ernet.in
}

\author{
V. Husna \\ Department of Mathematics, \\ Jnanabharathi Campus, \\ Bangalore University, India \\ email: husnav43@gmail.com, \\ husnav@bub.ernet.in
}

\begin{abstract}
In this paper, we discuss the problem of meromorphic functions sharing small function and present one theorem which extend a result of K. S. Charak and Banarasi Lal [16].
\end{abstract}

\section{Introduction and main results}

In this paper, a meromorphic function always mean a function which is meromorphic in the whole complex plane.

Definition 1 Let $\mathrm{f}(z)$ and $\mathrm{g}(z)$ be nonconstant meromorphic functions, a $\in$ $\mathbb{C} \cup\{\infty\}$. We say that $\mathrm{f}$ and $\mathrm{g}$ share the value a $C M$ if $\mathrm{f}-\mathrm{a}$ and $\mathrm{g}-\mathrm{a}$ have the same zeros with the same multiplicities.

Definition 2 We denote by $\mathrm{N}_{\mathrm{k}}\left(\mathrm{r}, \frac{1}{(\mathrm{f}-\mathrm{a})}\right)$ the counting function for zeros of $\mathrm{f}-\mathrm{a}$ with multiplicity $\leq \mathrm{k}$, and by $\overline{\mathrm{N}}_{\mathrm{k})}\left(\mathrm{r}, \frac{1}{(\mathrm{f}-\mathrm{a})}\right)$ the corresponding one for

2010 Mathematics Subject Classification: 30D35

Key words and phrases: meromorphic function, sharing values, differential monomial, polynomial 
which multiplicity is not counted. Let $\mathrm{N}_{(\mathrm{k}}\left(\mathrm{r}, \frac{1}{(\mathrm{f}-\mathrm{a})}\right)$ be the counting function for zeros of $\mathrm{f}-\mathrm{a}$ with multiplicity at least $\mathrm{k}$ and $\overline{\mathrm{N}}_{(\mathrm{k}}\left(\mathrm{r}, \frac{1}{(\mathrm{f}-\mathrm{a})}\right)$ the corresponding one for which multiplicity is not counted. Set

$\mathrm{N}_{k}\left(r, \frac{1}{f-a}\right)=\bar{N}\left(r, \frac{1}{f-a}\right)+\bar{N}_{(2}\left(r, \frac{1}{f-a}\right)+\ldots+\bar{N}_{(k}\left(r, \frac{1}{f-a}\right)$.

Definition 3 For two positive integers $n, p$ we define $\mu_{p}=\min \{n, p\}$ and $\mu_{\mathrm{p}}^{*}=\mathrm{p}+1-\mu_{\mathrm{p}}$. Then it is clear that

$$
N_{p}\left(r, 0 ; f^{n}\right) \leq \mu_{p} N_{\mu_{p}^{*}}(r, 0 ; f)
$$

Definition 4 [17] Let $z_{0}$ be a zero of $\mathrm{f}-\mathrm{a}$ of multiplicity $\mathrm{p}$ and a zero of $\mathrm{g}-\mathrm{a}$ of multiplicity $\mathrm{q}$. We denote by $\overline{\mathrm{N}}_{\mathrm{L}}(\mathrm{r}, \mathrm{a} ; \mathrm{f})$ the counting function of those a-points of $\mathrm{f}$ and $\mathrm{g}$ where $\mathrm{p}>\mathrm{q} \geq 1$, by $\mathrm{N}_{\mathrm{E}}^{1)}(\mathrm{r}, \mathrm{a} ; \mathrm{f})$ the counting function of those a-points of $\mathrm{f}$ and $\mathrm{g}$ where $\mathrm{p}=\mathrm{q}=1$ and by $\overline{\mathrm{N}}_{\mathrm{E}}^{(2}(\mathrm{r}, \mathrm{a} ; \mathrm{f})$ the counting function of those $\mathrm{a}$-points of $\mathrm{f}$ and $\mathrm{g}$ where $\mathrm{p}=\mathrm{q} \geq 2$, each point in these counting functions is counted only once. In the same way we can define $\bar{N}_{L}(r, a ; g), N_{E}^{1)}(r, a ; g), \bar{N}_{E}^{(2}(r, a ; g)$.

Definition 5 [18] Let $\mathrm{k}$ be a non-negative integer or infinity. For $\mathrm{a} \in \mathbb{C} \cup$ $\{\infty\}$ we denote by $\mathrm{E}_{\mathrm{k}}(\mathrm{a} ; \mathrm{f})$ the set of all a-points of $\mathrm{f}$, where an a-point of multiplicity $\mathrm{m}$ is counted $\mathrm{m}$ times if $\mathrm{m} \leq \mathrm{k}$ and $\mathrm{k}+1$ times if $\mathrm{m}>\mathrm{k}$. If $E_{k}(a ; f)=E_{k}(a ; g)$, we say that $f, g$ share the value a with weight $k$.

The definition implies that if $f, g$ share a value $a$ with weight $k$ then $z_{0}$ is an a-point of $f$ with multiplicity $m(\leq k)$ if and only if it is an a-point of $g$ with multiplicity $m(\leq k)$ and $z_{0}$ is an a-point of $f$ with multiplicity $m(>k)$ if and only if it is an a-point of $g$ with multiplicity $n(>k)$, where $m$ is not necessarily equal to $n$.

We write $f, g$ share $(a, k)$ to mean that $f, g$ share the value $a$ with weight $k$. Clearly if $f, g$ share $(a, k)$ then $f, g$ share $(a, p)$ for any integer $p, 0 \leq p<k$. Also we note that $f, g$ share a value $a$ IM or CM if and only if $f, g$ share $(a, 0)$ or $(a, \infty)$ respectively.

With the notion of weighted sharing of values Lahiri-Sarkar [13] improved the result of Zhang [14]. In [15] Zhang extended the result of Lahiri-Sarkar [13] and replaced the concept of value sharing by small function sharing.

In 2008, Zhang and Lü [12] obtained the following result. 
Theorem A Let $\mathrm{k}, \mathrm{n}$ be the positive integers, $\mathrm{f}$ be a non-constant meromorphic function, and $\mathrm{a}(\not \equiv 0, \infty)$ be a meromorphic function satisfying $\mathrm{T}(\mathrm{r}, \mathrm{a})=$ $\mathrm{o}(\mathrm{T}(\mathrm{r}, \mathrm{f}))$ as $\mathrm{r} \rightarrow \infty$. If $\mathrm{f}^{\mathrm{n}}$ and $\mathrm{f}^{(\mathrm{k})}$ share a IM and

$$
(2 k+6) \Theta(\infty, f)+4 \Theta(0, f)+2 \delta_{2+k}(0, f)>2 k+12-n,
$$

or $\mathrm{f}^{\mathrm{n}}$ and $\mathrm{f}^{(\mathrm{k})}$ share a $C M$ and

$$
(k+3) \Theta(\infty, f)+2 \Theta(0, f)+\delta_{2+k}(0, f)>k+6-n,
$$

then $\mathrm{f}^{\mathrm{n}}=\mathrm{f}^{(\mathrm{k})}$.

In the same paper, T. Zhang and W. Lü asked the following question:

Question 1 What will happen if $f^{n}$ and $\left(f^{(k)}\right)^{m}$ share a meromorphic function $\mathrm{a}(\not \equiv 0, \infty)$ satisfying $\mathrm{T}(\mathrm{r}, \mathrm{a})=\mathrm{o}(\mathrm{T}(\mathrm{r}, \mathrm{f}))$ as $r \rightarrow \infty$ ?

S. S. Bhoosnurmath and Kabbur [3] proved:

Theorem B Let $\mathrm{f}$ be a non-constant meromorphic function and $\mathrm{a}(\not \equiv 0, \infty)$ be a meromorphic function satisfying $\mathrm{T}(\mathrm{r}, \mathrm{a})=\mathrm{o}(\mathrm{T}(\mathrm{r}, \mathrm{f}))$ as $\mathrm{r} \rightarrow \infty$. Let $\mathrm{P}[\mathrm{f}]$ be a non-constant differential polynomial in $\mathrm{f}$. If $\mathrm{f}$ and $\mathrm{P}[\mathrm{f}]$ share a $I M$ and

$$
(2 \mathrm{Q}+6) \Theta(\infty, f)+(2+3 \underline{d}(P)) \delta(0, f)>2 Q+2 \underline{d}(P)+\bar{d}(P)+7,
$$

or if $\mathrm{f}$ and $\mathrm{P}[\mathrm{f}]$ share a $C M$ and

$$
3 \Theta(\infty, f)+(\underline{d}(P)+1) \delta(0, f)>4
$$

then $\mathrm{f} \equiv \mathrm{P}[\mathrm{f}]$.

Banerjee and Majumder [2] considered the weighted sharing of $f^{n}$ and $\left(f^{m}\right)^{(k)}$ and proved the following result:

Theorem $\mathbf{C}$ Let $\mathrm{f}$ be a non-constant meromorphic function, $\mathrm{k}, \mathrm{n}, \mathrm{m} \in \mathrm{N}$ and $\mathrm{l}$ be a non negative integer. Suppose $\mathrm{a}(\not \equiv 0, \infty)$ be a meromorphic function satisfying $\mathrm{T}(\mathrm{r}, \mathrm{a})=\mathrm{o}(\mathrm{T}(\mathrm{r}, \mathrm{f}))$ as $\mathrm{r} \rightarrow \infty$ such that $\mathrm{f}^{\mathrm{n}}$ and $\left(\mathrm{f}^{\mathrm{m}}\right)^{(\mathrm{k})}$ share $(\mathrm{a}, \mathrm{l})$. If $l \geq 2$ and

$$
(k+3) \Theta(\infty, f)+(k+4) \Theta(0, f)>2 k+7-n,
$$


or $l=1$ and

$$
\left(k+\frac{7}{2}\right) \Theta(\infty, f)+\left(k+\frac{9}{2}\right) \Theta(0, f)>2 k+8-n,
$$

or $l=0$ and

$$
(2 k+6) \Theta(\infty, f)+(2 k+7) \Theta(0, f)>4 k+13-n,
$$

then $f \equiv\left(f^{m}\right)^{(k)}$.

In 2015, Kuldeep S. Charak and Banarasi Lal [16] proved the following result:

Theorem $\mathbf{D}$ Let $\mathbf{f}$ be a non-constant meromorphic function, $\mathrm{n}$ be a positive integer and $\mathrm{a}(\not \equiv 0, \infty)$ be a meromorphic function satisfying $\mathrm{T}(\mathrm{r}, \mathrm{a})=\mathrm{o}(\mathrm{T}(\mathrm{r}, \mathrm{f}))$ as $\mathrm{r} \rightarrow \infty$. Let $\mathrm{P}[\mathrm{f}]$ be a non-constant differential polynomial in $\mathrm{f}$. Suppose $\mathrm{f}^{\mathrm{n}}$ and $\mathrm{P}[\mathrm{f}]$ share $(\mathrm{a}, \mathrm{l})$ such that any one of the following holds:

(i) when $l \geq 2$ and

$$
(Q+3) \Theta(\infty, f)+2 \Theta(0, f)+\bar{d}(P) \delta(0, f)>Q+5+2 \bar{d}(P)-\underline{d}(P)-n,
$$

(ii) when $\mathrm{l}=1$ and

$$
\left(\mathrm{Q}+\frac{7}{2}\right) \Theta(\infty, f)+\frac{5}{2} \Theta(0, f)+\bar{d}(P) \delta(0, f)>Q+6+2 \bar{d}(P)-\underline{d}(P)-n,
$$

(iii) when $l=0$ and

$(2 \mathrm{Q}+6) \Theta(\infty, f)+4 \Theta(0, f)+2 \overline{\mathrm{d}}(\mathrm{P}) \delta(0, f)>2 \mathrm{Q}+10+4 \overline{\mathrm{d}}(\mathrm{P})-2 \underline{\mathrm{d}}(\mathrm{P})-\mathrm{n}$.

Then $\mathrm{f}^{\mathrm{n}} \equiv \mathrm{P}[\mathrm{f}]$.

Through the paper we shall assume the following notations. Let

$$
\mathcal{P}(\omega)=a_{m+n} \omega^{m+n}+\ldots+a_{n} \omega^{n}+\ldots+a_{0}=a_{n+m} \prod_{i=1}^{s}\left(\omega-\omega_{p_{i}}\right)^{p_{i}}
$$

where $a_{j}(j=0,1,2, \ldots, n+m-1), a_{n+m} \neq 0$ and $\omega_{p_{i}}(i=1,2, \ldots, s)$ are distinct finite complex numbers and $2 \leq \mathrm{s} \leq \mathrm{n}+\mathrm{m}$ and $\mathrm{p}_{1}, \mathrm{p}_{2}, \ldots, \mathrm{p}_{\mathrm{s}}, \mathrm{s} \geq$ $2, n, m$ and $k$ are all positive integers with $\sum_{i=1}^{s} p_{i}=n+m$. Also let $p>$ $\max _{p \neq p_{i}, i=1, \ldots, r}\left\{p_{i}\right\}, r=s-1$, where $s$ and $r$ are two positive integers. 
Let

$$
P\left(\omega_{1}\right)=a_{n+m} \prod_{i=1}^{s-1}\left(\omega_{1}+\omega_{p}-\omega_{p_{i}}\right)^{p_{i}}=b_{q} \omega_{1}^{q}+b_{q-1} \omega_{1}^{q-1}+\ldots+b_{0},
$$

where $a_{n+m}=b_{q}, \omega_{1}=\omega-\omega_{p}, q=n+m-p$. Therefore, $\mathcal{P}(\omega)=\omega_{1}^{p} P\left(\omega_{1}\right)$. Next we assume

$$
P\left(\omega_{1}\right)=b_{q} \prod_{i=1}^{r}\left(\omega_{1}-\alpha_{i}\right)^{p_{i}},
$$

where $\alpha_{i}=\omega_{p_{i}}-\omega_{p},(i=1,2, \ldots, r)$, be distinct zeros of $P\left(\omega_{1}\right)$.

In this paper we will prove one theorem which will improve and generalize Theorem D.

Theorem 1 Let $\mathrm{k}(\geq 1), \mathrm{n}(\geq 1), \mathrm{p}(\geq 1)$ and $\mathrm{m}(\geq 0)$ be integers and $\mathrm{f}$ and $\mathrm{f}_{1}=$ $\mathrm{f}-\omega_{\mathrm{p}}$ be two nonconstant meromorphic functions and $\mathrm{M}[\mathrm{f}]$ be a differential monomial of degree $\mathrm{d}_{\mathrm{M}}$ and weight $\Gamma_{\mathrm{M}}$ and $\mathrm{k}$ is the highest derivative in $\mathrm{M}[\mathrm{f}]$. Let $\mathcal{P}(z)=a_{m+n} z^{m+n}+\ldots+a_{n} z^{n}+\ldots+a_{0}, a_{m+n} \neq 0$, be a polynomial in $z$ of degree $m+n$ such that $\mathcal{P}(\mathbf{f})=\mathrm{f}_{1}^{p} \mathrm{P}\left(\mathrm{f}_{1}\right)$. Also let $\mathrm{a}(z)(\not \equiv 0, \infty)$ be a small function with respect to $\mathrm{f}$. Suppose $\mathcal{P}(\mathrm{f})-\mathrm{a}$ and $\mathrm{M}[\mathrm{f}]-\mathrm{a}$ share $(0, \mathrm{l})$. If $\mathrm{l} \geq 2$ and

$$
(3+2 \lambda) \Theta(\infty, f)+\mu_{2} \delta_{\mu_{2}^{*}}\left(\omega_{p}, f\right)+2 d_{M} \delta_{1+k}(0, f)>2 \Gamma_{M}+2 \mu_{2}+3-p
$$

or $l=1$ and

$$
\begin{gathered}
\left(\frac{7}{2}+2 \lambda\right) \Theta(\infty, f)+\frac{1}{2} \Theta\left(\omega_{p}, f\right)+\mu_{2} \delta_{\mu_{2}^{*}}\left(\omega_{p}, f\right)+2 d_{M} \delta_{1+k}(0, f)> \\
2 \Gamma_{M}+\mu_{2}+4+\frac{(m+n)-3 p}{2}
\end{gathered}
$$

or $l=0$ and

$$
\begin{aligned}
(6+3 \lambda) \Theta(\infty, f) & +2 \Theta\left(\omega_{p}, f\right)+\mu_{2} \delta_{\mu_{2}^{*}}\left(\omega_{p}, f\right)+3 d_{M} \delta_{1+k}(0, f) \\
& >3 \Gamma_{M}+\mu_{2}+8+2(m+n)-3 p
\end{aligned}
$$

then $\mathcal{P}(f) \equiv M[f]$.

Following example shows that in Theorem $1 \mathrm{a}(z) \not \equiv 0$ is essential.

Example 1 Let us take $\mathrm{f}(\mathrm{z})=\mathrm{e}^{\mathrm{L} z}$ where $\mathrm{L} \neq 0, \pm 1$ and $\mathcal{P}(\mathrm{f})=\mathrm{f}^{3}, \mathrm{M}[\mathrm{f}]=\mathrm{f}^{(2)}$. Then $\mathcal{P}(\mathrm{f})$ and $\mathrm{M}[\mathrm{f}]$ share $\mathrm{a}=0$ (or, $\infty)$. Here $\mathrm{m}=0, \mathrm{p}=\mathrm{n}=1, \omega_{\mathrm{p}}=$ $0, \mathrm{~d}_{M}=1, \mu_{2}=1, \Gamma_{M}=3$ and $\lambda=2$. Also $\Theta(\infty ; f)=1=\Theta(0 ; f)$ and $\delta_{\mathrm{p}}(0 ; f)=1, \forall \mathrm{q} \in \mathbb{N}$. Thus we see that the deficiency conditions stated in Theorem 1 are satisfied but $\mathcal{P}(\mathrm{f}) \not \equiv \mathrm{M}[\mathrm{f}]$. 
The next example shows that the deficiency conditions stated in Theorem 1 are not necessary.

Example 2 Let $\mathrm{f}(z)=\mathrm{C} \cos z+D \sin z, C D \neq 0$. Then $\bar{N}(r, f)=S(r, f)$ and

$$
\bar{N}(r, 0 ; f)=\bar{N}\left(r, \frac{C+i D}{C-i D} ; e^{2 i z}\right) \sim T(r, f) .
$$

Here $\mathrm{m}=0, \mathrm{p}=\mathrm{n}=1, \omega_{\mathrm{p}}=0, \mathrm{~d}_{\mathrm{M}}=1, \mu_{2}=1, \Gamma_{\mathrm{M}}=4 \mathrm{k}+1$ and $\lambda=4 \mathrm{k}$. Again $\Theta(\infty, f)=1$ and $\Theta(0, f)=\delta_{p}(0, f)=0$. Let $\mathrm{m}=0$, hence $\mathcal{P}(\mathrm{f})=\mathrm{f}$.

Therefore it is clear that $\mathrm{M}[\mathrm{f}]=\mathrm{f}^{(4 \mathrm{k})}$, for $\mathrm{k} \in \mathbb{N}$ and $\mathcal{P}(\mathrm{f})$ share $\mathrm{a}(z)$ and the deficiency conditions in Theorem 1 are not satisfied, but $\mathcal{P}(\mathrm{f}) \equiv \mathrm{M}$.

\section{Lemmas}

Lemma 1 [17] For the differential monomial $\mathrm{M}[\mathrm{f}]$,

$$
N_{p}(r, 0 ; M[f]) \leq d_{M} N_{p+k}(r, 0 ; g)+\lambda \bar{N}(r, \infty, f)+S(r, f) .
$$

Lemma 2 [17] Let $\mathrm{F}$ and $\mathrm{G}$ share $(1, \mathrm{l})$. Then

$$
\bar{N}_{L}(r, 1 ; F) \leq \frac{1}{l+1} \bar{N}(r, \infty ; F)+\frac{1}{l+1} \bar{N}(r, 0 ; F)+S(r, F) \text { if } l \geq 1,
$$

and

$$
\overline{\mathrm{N}}_{\mathrm{L}}(\mathrm{r}, 1 ; \mathrm{F}) \leq \overline{\mathrm{N}}(\mathrm{r}, \infty ; \mathrm{F})+\overline{\mathrm{N}}(\mathrm{r}, 0 ; \mathrm{F})+\mathrm{S}(\mathrm{r}, \mathrm{F}) \text { if } \mathrm{l}=0 .
$$

Lemma 3 Let $\mathrm{f}$ be a non-constant meromorphic function and $\mathrm{a}(\mathrm{z})$ be a small function of $\mathrm{f}$. Let us define $\mathrm{F}=\frac{\mathcal{P}(\mathrm{f})}{\mathrm{a}}=\frac{\mathrm{f}_{1}^{\mathrm{p}} \mathrm{P}\left(\mathrm{f}_{1}\right)}{\mathrm{a}}$ and $\mathrm{G}=\frac{\mathrm{M}[\mathrm{f}]}{\mathrm{a}}$. Then $\mathrm{FG} \not \equiv 1$. Proof. On contrary suppose $\mathrm{FG} \equiv 1$ i.e

$$
f_{1}^{p} P\left(f_{1}\right) M[f]=a^{2} .
$$

From above it is clear that the function $f$ can't have any zero and poles. Therefore $\bar{N}(r, 0 ; f)=S(r, f)=\bar{N}(r, \infty ; f)$. So by the First Fundamental Theorem and Lemma 1, we have

$$
\begin{aligned}
\left(m+n+d_{M}\right) T(r, f) & =T\left(r, \frac{a^{2}}{f_{1}^{p} P\left(f_{1}\right) f^{d_{M}}}\right)+S(r, f) \leq T\left(r, \frac{M[f]}{f^{d_{M}}}\right)+S(r, f) \\
& \leq m\left(r, \frac{M[f]}{f^{d_{M}}}\right)+N\left(r, \frac{M[f]}{f^{d_{M}}}\right)+S(r, f) \\
& \leq N\left(r, \frac{M[f]}{f^{d_{M}}}\right)+S(r, f)
\end{aligned}
$$


Then using Lemma 2 and from above inequality, we get

$$
\left(m+n+d_{M}\right) T(r, f) \leq d_{M} N(r, 0 ; f)+\lambda \bar{N}(r, f)+S(r, f) \leq S(r, f),
$$

which is not possible.

Lemma 4 [17] Let $\mathrm{f}$ be a non-constant meromorphic function and $\mathrm{a}(\mathrm{z})$ be a small function of $\mathrm{f}$. Let $\mathrm{F}=\frac{\mathcal{P}(\mathrm{f})}{\mathrm{a}}=\frac{\mathrm{f}_{1}^{\mathrm{p}} \mathrm{P}\left(\mathrm{f}_{1}\right)}{\mathrm{a}}$ and $\mathrm{G}=\frac{\mathrm{M}[\mathrm{f}]}{\mathrm{a}}$ such that $\mathrm{F}$ and $\mathrm{G}$ shares $(1, \infty)$. Then one of the following cases holds:

1. $T(r) \leq N_{2}(r, 0 ; F)+N_{2}(r, 0 ; G)+\bar{N}(r, \infty ; F)+\bar{N}(r, \infty ; G)+\bar{N}_{L}(r, \infty ; F)+$ $\overline{\mathrm{N}}_{\mathrm{L}}(\mathrm{r}, \infty ; \mathrm{G})+\mathrm{S}(\mathrm{r})$,

2. $F \equiv G$,

3. $\mathrm{FG} \equiv 1$.

where $\mathrm{T}(\mathrm{r})=\max \{\mathrm{T}(\mathrm{r}, \mathrm{F}), \mathrm{T}(\mathrm{r}, \mathrm{G})\}$ and $\mathrm{S}(\mathrm{r})=\mathrm{o}(\mathrm{T}(\mathrm{r})), \mathrm{r} \in \mathrm{I}$, I is a set of infinite linear measure of $r \in\{0, \infty\}$.

\section{Proof of the Theorem}

\section{Proof.}

Let $F=\frac{\mathcal{P}(f)}{a}=\frac{f_{1}^{p} P\left(f_{1}\right)}{a}$ and $G=\frac{M[f]}{a}$. Then $F-1=\frac{f_{1}^{p} P\left(f_{1}\right)-a}{a}$ and $G-1=$ $\frac{M[f]-a}{a}$. Since $\mathcal{P}(f)$ and $M[f]$ share $(a, l)$, it follows that $F$ and $G$ share $(1, l)$, except the zeros and poles of $a(z)$.

Define

$$
\psi=\left(\frac{F^{\prime \prime}}{F^{\prime}}-\frac{2 F^{\prime}}{F-1}\right)-\left(\frac{G^{\prime \prime}}{G^{\prime}}-\frac{2 G^{\prime}}{G-1}\right) .
$$

We consider the following cases:

Case 1. When $\psi \not \equiv 0$. Then from (4), we have $m(r, \psi)=S(r, f)$. By the second fundamental theorem of Nevanlinna, we have

$$
\begin{aligned}
T(r, F)+T(r, G) & \leq 2 \bar{N}(r, f)+\bar{N}\left(r, \frac{1}{F}\right)+\bar{N}\left(r, \frac{1}{F-1}\right)+\bar{N}\left(r, \frac{1}{G}\right) \\
& +\bar{N}\left(r, \frac{1}{G-1}\right)-N_{0}\left(r, \frac{1}{F^{\prime}}\right)-N_{0}\left(r, \frac{1}{G^{\prime}}\right)+S(r, f),
\end{aligned}
$$

where $N_{0}\left(r, \frac{1}{F^{\prime}}\right)$ denotes the counting function of the zeros of $F^{\prime}$ which are not the zeros of $F(F-1)$ and $N_{0}\left(r, \frac{1}{G^{\prime}}\right)$ denotes the counting function of the zeros 
of $G^{\prime}$ which are not the zeros of $G(G-1)$.

Subcase 1.1. When $l \geq 1$. Then from (4), we have,

$$
\begin{aligned}
N_{E}^{1)}\left(r, \frac{1}{F-1}\right) \leq & N\left(r, \frac{1}{\psi}\right)+S(r, f) \leq T(r, \psi)+S(r, f)=N(r, \psi)+S(r, f) \\
\leq & \bar{N}(r, F)+\bar{N}_{(2}\left(r, \frac{1}{F}\right)+\bar{N}_{(2}\left(r, \frac{1}{G}\right)+\bar{N}_{L}\left(r, \frac{1}{F-1}\right) \\
& +\bar{N}_{L}\left(r, \frac{1}{G-1}\right)+N_{0}\left(r, \frac{1}{F^{\prime}}\right)+N_{0}\left(r, \frac{1}{G^{\prime}}\right)+S(r, f)
\end{aligned}
$$

and so

$$
\begin{aligned}
& \bar{N}\left(r, \frac{1}{F-1}\right)+\bar{N}\left(r, \frac{1}{G-1}\right)=N_{E}^{1)}\left(r, \frac{1}{F-1}\right)+\bar{N}_{E}^{(2}\left(r, \frac{1}{F-1}\right) \\
& \quad+\bar{N}_{L}\left(r, \frac{1}{F-1}\right)+\bar{N}_{L}\left(r, \frac{1}{G-1}\right)+\bar{N}\left(r, \frac{1}{G-1}\right)+S(r, f) \\
& \leq \bar{N}(r, f)+\bar{N}_{(2}\left(r, \frac{1}{\bar{F}}\right)+\bar{N}_{(2}\left(r, \frac{1}{G}\right)+2 \bar{N}_{L}\left(r, \frac{1}{F-1}\right)+2 \bar{N}_{L}\left(r, \frac{1}{G-1}\right) \\
& \quad+\bar{N}_{E}^{(2}\left(r, \frac{1}{F-1}\right)+\bar{N}\left(r, \frac{1}{G-1}\right)+N_{O}\left(r, \frac{1}{F^{\prime}}\right)+N_{O}\left(r, \frac{1}{G^{\prime}}\right)+S(r, f) .
\end{aligned}
$$

For $l \geq 2$, we have

$$
\begin{aligned}
& 2 \bar{N}_{L}\left(r, \frac{1}{F-1}\right)+2 \bar{N}_{L}\left(r, \frac{1}{G-1}\right)+\bar{N}_{E}^{(2}\left(r, \frac{1}{F-1}\right)+\bar{N}\left(r, \frac{1}{G-1}\right) \\
& \leq N\left(r, \frac{1}{G-1}\right)+S(r, f) .
\end{aligned}
$$

Thus from (6), we obtain

$$
\begin{aligned}
& \bar{N}\left(r, \frac{1}{F-1}\right)+\bar{N}\left(r, \frac{1}{G-1}\right) \leq \bar{N}(r, f)+\bar{N}_{(2}\left(r, \frac{1}{F}\right) \\
& +\bar{N}_{(2}\left(r, \frac{1}{G}\right)+N\left(r, \frac{1}{G-1}\right)+N_{0}\left(r, \frac{1}{F^{\prime}}\right)+N_{0}\left(r, \frac{1}{G^{\prime}}\right)+S(r, f) .
\end{aligned}
$$


Now from Lemma 1, (5) and (7) we obtain

$$
\begin{aligned}
& T(r, F) \leq 3 \bar{N}(r, f)+\bar{N}\left(r, \frac{1}{\bar{F}}\right)+\bar{N}_{(2}\left(r, \frac{1}{\bar{F}}\right)+\bar{N}\left(r, \frac{1}{G}\right)+\bar{N}_{(2}\left(r, \frac{1}{G}\right)+S(r, f) \\
& \leq 3 \bar{N}(r, f)+2 \bar{N}\left(r, \frac{1}{\bar{F}}\right)+N\left(r, \frac{1}{G}\right)+S(r, f) \\
& \leq 3 \bar{N}(r, f)+\mu_{2} N_{\mu_{2}^{*}}\left(r, \omega_{p} ; f\right)+2 d_{M} N_{1+k}\left(r, \frac{1}{f}\right)+2 \lambda \bar{N}(r, f)+S(r, f) \\
&(n+m) T(r, f) \leq(3+2 \lambda) \bar{N}(r, f)+\mu_{2} N_{\mu_{2}^{*}}\left(r, \omega_{p} ; f\right)+(m+n-p) T(r, f)+2 d_{M} N_{1+k}\left(r, \frac{1}{f}\right)+S(r, f) \\
&\left\{(3+2 \lambda) \Theta(\infty, f)+\mu_{2} \delta_{\mu_{2}^{*}}\left(r, \omega_{p} ; f\right)+2 d_{M} \delta_{1+k}(0, f)\right\} T(r, f) \\
& \leq\left(3+2 \lambda+2 \mu_{1}+m_{1}+n-2 p+2 d_{M}\right) T(r, f)+S(r, f)
\end{aligned}
$$

which violates (1).

Next, consider the case when $l=1$.

First note that

$$
\overline{\mathrm{N}}_{\mathrm{L}}\left(\mathrm{r}, \frac{1}{\mathrm{~F}-1}\right) \leq \frac{1}{2} \mathrm{~N}\left(\mathrm{r}, \frac{1}{\mathrm{~F}^{\prime}} \mid \mathrm{F} \neq 0\right) \leq \frac{1}{2} \overline{\mathrm{N}}(\mathrm{r}, \mathrm{F})+\frac{1}{2} \overline{\mathrm{N}}\left(\mathrm{r}, \frac{1}{\mathrm{~F}}\right),
$$

when $N\left(r, \frac{1}{F^{\prime}} \mid F \neq 0\right)$ denotes the zeros of $F^{\prime}$, that are not the zeros of $F$. From (4) and (8), we have

$$
\begin{aligned}
2 \bar{N}_{L}\left(r, \frac{1}{F-1}\right) & +2 \bar{N}_{L}\left(r, \frac{1}{F-1}\right)+\bar{N}_{E}^{(2}\left(r, \frac{1}{F-1}\right)+\bar{N}\left(r, \frac{1}{G-1}\right) \\
& \leq N\left(r, \frac{1}{G-1}\right)+\bar{N}_{L}\left(r, \frac{1}{F-1}\right)+S(r, f) \\
& \leq N\left(r, \frac{1}{G-1}\right)+\frac{1}{2} \bar{N}(r, F)+\frac{1}{2} \bar{N}\left(r, \frac{1}{F}\right)+S(r, f)
\end{aligned}
$$


Thus, from (5) and (9), we have

$$
\begin{aligned}
& \bar{N}\left(r, \frac{1}{F-1}\right)+\bar{N}\left(r, \frac{1}{G-1}\right) \leq \bar{N}(r, f)+\bar{N}_{(2}\left(r, \frac{1}{F}\right)+\bar{N}_{(2}\left(r, \frac{1}{G}\right) \\
& +\frac{1}{2} \bar{N}(r, F)+\frac{1}{2} \bar{N}\left(r, \frac{1}{F}\right)+T(r, G)+N_{0}\left(r, \frac{1}{F^{\prime}}\right)+N_{0}\left(r, \frac{1}{G^{\prime}}\right)+S(r, f) .
\end{aligned}
$$

From Lemma 1, (5) and (10) we obtain

$$
\begin{aligned}
& T(r, F) \leq 3 \bar{N}(r, F)+\bar{N}\left(r, \frac{1}{F}\right)+\bar{N}_{(2}\left(r, \frac{1}{F}\right)+\bar{N}\left(r, \frac{1}{G}\right)+\bar{N}_{(2}\left(r, \frac{1}{G}\right) \\
&+\frac{1}{2} \bar{N}(r, F)+\frac{1}{2} \bar{N}\left(r, \frac{1}{F}\right)+S(r, f) \\
& \leq \frac{7}{2} \bar{N}(r, f)+2 \bar{N}\left(r, \frac{1}{F}\right)+2 \bar{N}\left(r, \frac{1}{G}\right)+\frac{1}{2} \bar{N}\left(r, \frac{1}{F}\right)+S(r, f) \\
& \leq \frac{7}{2} \bar{N}(r, f)+\mu_{2} N_{\mu_{2}^{*}}\left(r, \omega_{p} ; f\right)+(m+n-p) T(r, f)+2 d_{M} N_{1+k}\left(r, \frac{1}{f}\right) \\
&+2 \lambda \bar{N}(r, f)+\frac{1}{2}\left\{\bar{N}\left(r, \omega_{p} ; f\right)+(m+n-p) T(r, f)+S(r, f)\right\} \\
&(m+n) T(r, f) \leq\left(\frac{7}{2}+2 \lambda\right)(1-\Theta(\infty, f))+\mu_{2}\left(1-\delta_{\mu_{2}^{*}}\left(\omega_{p}, f\right)\right)+\frac{3}{2}(m+n-p) \\
&+2 d_{M}\left(1-\delta_{1+k}(0, f)\right)+\frac{1}{2}\left(1-\Theta\left(\omega_{p}, f\right)\right)+S(r, f) . \\
&\left.\left\{\left(\frac{7}{2}+2 \lambda\right) \Theta(\infty, f)+\mu_{2} \delta_{\mu_{2}^{*}}\left(\omega_{p}, f\right)\right)+2 d_{M} \delta_{1+k}(0, f)+\frac{1}{2} \Theta\left(\omega_{p} ; f\right)\right\} \\
& \leq\left(\frac{7}{2}+2 \lambda+\mu_{2}+\frac{3}{2}(m+n-p)+2 d_{M}+\frac{1}{2}-m-n+\epsilon\right) T(r, f)+S(r, f) \\
& \leq\left(2 \Gamma_{M}+4+\mu_{2}+\frac{1}{2} m+\frac{1}{2} n-\frac{3}{2} p\right) T(r, f)+S(r, f)
\end{aligned}
$$

which violates (2).

Subcase 1.2. When $l=0$. Then, we have

$$
\begin{aligned}
& N_{E}^{1)}\left(r, \frac{1}{F-1}\right)=N_{E}^{1)}\left(r, \frac{1}{G-1}\right)+S(r, f), \\
& \bar{N}_{E}^{(2}\left(r, \frac{1}{F-1}\right)=\bar{N}_{E}^{(2}\left(r, \frac{1}{G-1}\right)+S(r, f),
\end{aligned}
$$


and also from (4), we have

$$
\begin{aligned}
& \bar{N}\left(r, \frac{1}{F-1}\right)+\bar{N}\left(r, \frac{1}{G-1}\right) \leq N_{E}^{1)}\left(r, \frac{1}{F-1}\right)+\bar{N}_{E}^{(2}\left(r, \frac{1}{F-1}\right) \\
& \quad+\bar{N}_{L}\left(r, \frac{1}{F-1}\right)+\bar{N}_{L}\left(r, \frac{1}{G-1}\right)+\bar{N}\left(r, \frac{1}{G-1}\right)+S(r, f) \\
& \leq N_{E}^{1)}\left(r, \frac{1}{F-1}\right)+\bar{N}_{L}\left(r, \frac{1}{F-1}\right)+N\left(r, \frac{1}{G-1}\right)+S(r, f) \\
& \leq \bar{N}(r, F)+\bar{N}_{(2}\left(r, \frac{1}{F}\right)+\bar{N}_{(2}\left(r, \frac{1}{G}\right)+2 \bar{N}_{L}\left(r, \frac{1}{F-1}\right)+\bar{N}_{L}\left(r, \frac{1}{G-1}\right) \\
& +N\left(r, \frac{1}{G-1}\right)+N_{O}\left(r, \frac{1}{F^{\prime}}\right)+N_{O}\left(r, \frac{1}{G^{\prime}}\right)+S(r, f)
\end{aligned}
$$

From Lemma 2, (5) and (9), we obtain

$$
\begin{aligned}
& T(r, F) \leq 3 \bar{N}(r, F)+\bar{N}\left(r, \frac{1}{F}\right)+\bar{N}_{(2}\left(r, \frac{1}{F}\right)+\bar{N}\left(r, \frac{1}{G}\right)+\bar{N}_{(2}\left(r, \frac{1}{G}\right) \\
&+2 \bar{N}_{L}\left(r, \frac{1}{F-1}\right)+\bar{N}_{L}\left(r, \frac{1}{G-1}\right)+S(r, f) \\
& \leq 6 \bar{N}(r, f)+\mu_{2} N_{\mu_{2}^{*}}(r, 0 ; f)+(m+n-p) T(r, f)+3\left(d_{M} N_{1+k}(r, 0 ; f)\right. \\
&+\lambda \bar{N}(r, f))+2\left\{\bar{N}\left(r, \omega_{p} ; f\right)+(m+n-p) T(r, f)\right\}+S(r, f) \\
&(m+n) T(r, f) \leq(6+3 \lambda)(1-\Theta(\infty, f))+\mu_{2}\left(1-\delta_{\mu_{2}^{*}}(r, f)\right)+3(m+n-p) \\
&+3 d_{M}\left(1-\delta_{1+k}(0, f)\right)+2\left(1-\Theta\left(\omega_{p}, f\right)\right)+S(r, f) . \\
&\left\{(6+3 \lambda) \Theta(\infty, f)+\mu_{2} \delta_{\mu_{2}^{*}}(r, f)+3 d_{M} \delta_{1+k}(0, f)+2 \Theta\left(\omega_{p}, f\right)-\epsilon\right\} T(r, f) \\
& \leq\left(6+3 \lambda+\mu_{2}+3 m+3 n-3 p+3 d_{M}+2-m-n\right) T(r, f)+S(r, f) \\
& \leq\left(3 \Gamma_{M}+\mu_{2}+2 m+2 n-3 p+8-\epsilon\right) T(r, f)+S(r, f)
\end{aligned}
$$

which violates (3).

Case 2. Let $\mathrm{H} \equiv 0$.

On Integration we get

$$
\frac{1}{G-1} \equiv \frac{A}{F-1}+B,
$$

where $A(\neq 0), B$ are complex constants.

It is clear that $F$ and $G$ share $(1, \infty)$. Also by construction of $F$ and $G$ we see that $F$ and $G$ share $(\infty, 0)$ also. 
So using Lemma 1 and condition (2), we obtain

$$
\begin{aligned}
N_{2} & (r, 0 ; F)+N_{2}(r, 0 ; G)+\bar{N}(r, \infty ; F)+\bar{N}(r, \infty ; G)+\bar{N}_{L}(r, \infty ; F) \\
& +\bar{N}_{L}(r, \infty ; G)+S(r) \leq 2 \bar{N}(r, 0 ; F)+2 \bar{N}(r, 0 ; G)+3 \bar{N}(r, \infty ; f)+S(r) \\
\leq & 2\left(\bar{N}\left(r, \omega_{p} ; f\right)+(m+n-p) T(r, f)\right)+2\left(d_{M} N_{1+k}\left(r, \frac{1}{f}\right)+\lambda \bar{N}(r, f)\right) \\
& +3 \bar{N}(r, f)+S(r) \leq 2\left(1-\Theta\left(\omega_{p}, f\right)\right)+2 d_{M}\left(1-\delta_{1+k}(0, f)\right) \\
& +(3+2 \lambda)(1-\Theta(\infty, f))+S(r)+(m+n-p) T(r, f) \\
\leq & \left(3+2 \lambda+2 d_{M}+2+m+n-p\right)-\left(3+2 \lambda+2 d_{M}+2-p\right) T(r, f)+S(r) \\
\leq & (m+n) T(r, f)+S(r)<T(r, F)+S(r) .
\end{aligned}
$$

Hence inequality (1) of Lemma 4 does not hold. Again in view of Lemma 3, we get $F G \not \equiv 1$. Therefore $F \equiv G$ i.e., $\mathcal{P}(f) \equiv M[f]$.

\section{Acknowledgement}

The author (VH) is greatful to the University Grants Commission(UGC), New Delhi, India for supporting her research work by providing her with a Maulana Azad National Fellowship(MANF).

\section{References}

[1] Banerjee, Abhijit, Meromorphic functions sharing one value. Int. J. Math. Math. Sci., 22 (2005), 3587-3598.

[2] A. Banerjee, S. Majumder, Some uniqueness results related to meromorphic function that share a small function with its derivative. Math. Rep. (Bucur.), 16 (66) (2014), no. 1, 95-111.

[3] Subhas S. Bhoosnurmath, Smita R. Kabbur, On entire and meromorphic functions that share one small function with their differential polynomial, Int. J. Anal., (2013), Art. ID 926340, 8 pp.

[4] S. S. Bhoosnurmath, Anupama J. Patil, On the growth and value distribution of meromorphic functions and their differential polynomials, $J$. Indian Math. Soc. (N.S.), 74 (2007), no. 3-4, 167-184 (2008). 
[5] W. K. Hayman, Meromorphic functions, Oxford Mathematical Monographs Clarendon Press, Oxford 1964 xiv+191 pp.

[6] H. -X. Yi, Uniqueness of meromorphic functions and a question of C. C. Yang. Complex Var. Theory and Appl., 14 (1990), no. 1-4, 169-176.

[7] H. -X. Yi, Uniqueness theorems for meromorphic functions whose nth derivatives share the same 1-points, Complex Var. Theory and Appl., 34 (1997), no. 4, 421-436.

[8] H. Huang, B. Huang, Uniqueness of meromorphic functions concerning differential monomials, Appl. Math. (Irvine), 2 (2011), no. 2, 230-235.

[9] N. Li, L.-Z. Yang, , Meromorphic function that shares one small function with its differential polynomial, Kyungpook Math. J., 50 (2010), no. 3, $447-454$.

[10] E. Mues, N. Steinmetz, Meromorphe Funktionen, die mit ihrer Ableitung Werte teilen. (German) Manuscripta Math., 29 (1979), no. 2-4, 195-206.

[11] C.-C. Yang, H.-X. Yi, Uniqueness theory of meromorphic functions. Mathematics and its Applications, 557. Kluwer Academic Publishers Group, Dordrecht, 2003. viii+569 pp. ISBN: 1-4020-1448-1

[12] T. Zhang, W. Lü, Notes on a meromorphic function sharing one small function with its derivative, Complex Var. Elliptic Equ., 53 (2008), no. 9, 857-867.

[13] I. Lahiri, A. Sarkar, Uniqueness of a meromorphic function and its derivative, JIPAM. J. Inequal. Pure Appl. Math., 5 (2004), no. 1, Article 20, 9 pp. (electronic).

[14] Zhang, Qing Cai, The uniqueness of meromorphic functions with their derivatives, Kodai Math. J., 21 (1998), no. 2, 179-184.

[15] Q. C. Zhang, Meromorphic function that shares one small function with its derivative, J. Inequal. Pure. Appl. Math., 6 (2015),1-13.

[16] K. S. Charak, Banarsi Lal, Uniqueness of $f^{n}$ and $P[f]$, arXiv:1501.05092v1.[math.CV]21 Jan 2015.

[17] A. Banerjee, B. Chakraborty, Further investigations on a question of Zhang and Lü, Ann. Univ. Paedagog. Crac. Stud. Math., 14 (2015), 105119. 
[18] A. Banerjee, S. Majumder, Sujoy, On the uniqueness of a power of a meromorphic function sharing a small function with the power of its derivative, Comment. Math. Univ. Carolin., 51 (2010), no. 4, 565-576.

[19] I. Lahiri, Weighted value sharing and uniqueness of meromorphic functions, Complex Var. Theory and Appl., 46 (2001), no. 3, 241-253. 